\title{
AIR BRONCHOGRAM: THE UNTRUSTWORTHY SIGN
}

\section{Qurat-Ul-Ain Shah, Uneza Masood, Imran K. Niazi}

Department of Radiology, Shaukat Khanum Memorial Cancer Hospital and Research Centre, Lahore, Pakistan

Received: 06 June 2018 / Accepted 13 June 2018

A 49-year-old postmenopausal lady presented with a history of painless right breast lump observed a month ago.

The patient underwent routine radiological investigation that confirmed malignant lesion in upper outer quadrant of the right breast with satellite nodule and pathological right axillary lymphadenopathy [Figure 1].

Routine pre-operative chest radiograph showed opacities in mid-lung zone bilaterally which were further evaluated with contrast-enhanced computed tomography (CT) thorax.

CT chest demonstrated consolidation with air bronchogram sign in bilateral perihilar hilar regions and similar opacity at the right lung base [Figure 2]. Possibility of pulmonary infection was given. However, considering dense appearance of the consolidations biopsy of the lesion was recommended in multidisciplinary meeting for final tissue diagnosis.

CT-guided fine-needle aspiration cytology of the left lung consolidation confirmed pulmonary metastasis and patient's treatment for breast cancer with pulmonary metastasis was commenced.

Breast cancer is the most prevalent cancer and the primary cause of cancer death in women. The mortality is not due to the primary tumour itself but is secondary of metastasis. Therefore, the detection of breast cancer metastasis at the earliest stage is important for the management and help to determine the prognosis. ${ }^{[1]}$ The lung is the common site from metastasis from breast cancer. Presentation can be with solitary or multiple pulmonary nodules, lymphangitic carcinomatosis and endobronchial metastasis being common manifestations. However, involvement as airspace consolidation is rare. Therefore, we report a case of breast cancer metastasized to lung that presented as bilateral perihilar dense consolidations with air bronchogram sign. ${ }^{[2,3]}$

Correspondence: Dr. Qurat-Ul-Ain Shah,

Department of Radiology, Shaukat Khanum Memorial Cancer

Hospital and Research Centre, Lahore, Pakistan.

Email: drshah218@gmail.com

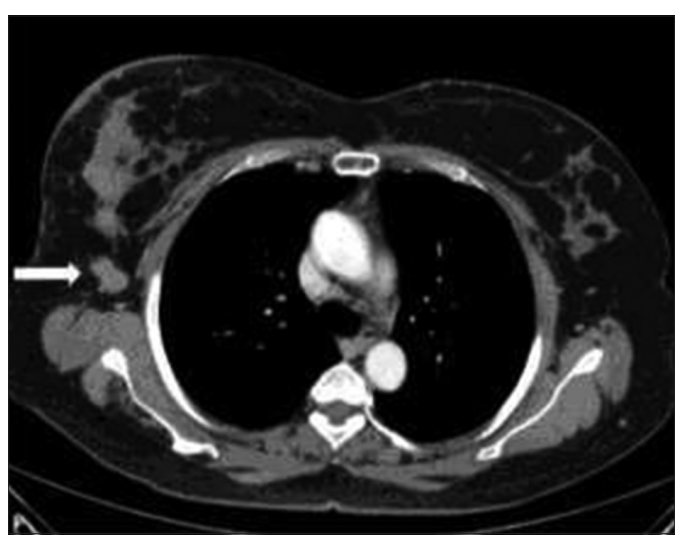

Figure 1: The right breast neoplastic lesion with pathological right axillary lymphadenopathy

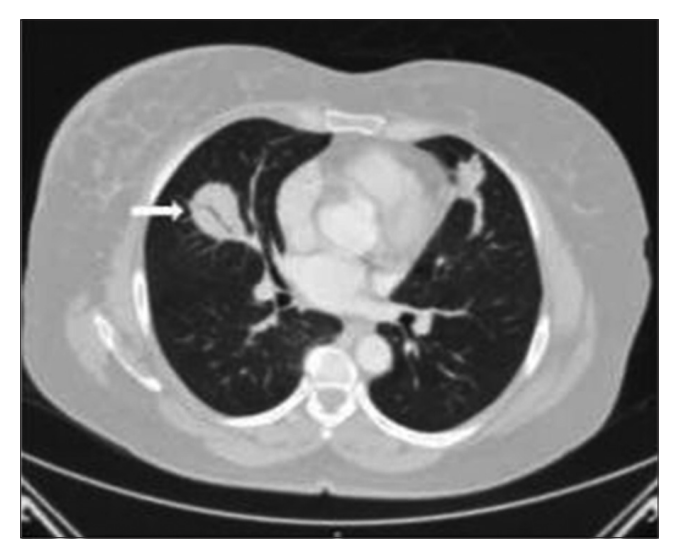

Figure 2: Bilateral perihilar consolidations with air bronchograms

\section{Conflict of Interest}

The authors declare that they have no conflict of interest.

\section{References}

1. Scully OJ, Bay BH, Yip G, et al. Breast cancer metastasis. Cancer Genomics Proteomics 2012;9:311-20.

2. Ohnishi H, Haruta Y, Yokoyama A, et al. Metastatic breast cancer presenting as air-space consolidation on chest computed tomography. Intern Med 2009;48:727-31.

3. Kim SB, Lee S, Koh MJ, et al. Ground-glass opacity in lung metastasis from breast cancer: A case report. Tuberc Respir Dis (Seoul) 2013;74:32-6. 